\title{
Argumentación e INCERTIDUMBre. ¿Es POSIBLE UNA teORÍA UNIFICADA DE LA ARGUMENTACIÓN?
}

Mario Gensollen

Departamento de Filosofía, Universidad Autónoma de Aguascalientes, México

mgenso@gmail.com

Resumen

El objetivo de este artículo es indagar cuáles son los elementos que se requieren para una teoría unificada de la argumentación, dado que ésta podría ser una respuesta ante las insinuaciones relativistas, que se acompañan de la sofística del todo o nada. Se propone considerar diversos elementos para dicho objetivo, tales como agente, acción, consecuencias de la acción, contexto y el concepto de "práctica".

Palabras clave: agente argumentativo, argumentación, Carlos Pereda, práctica argumentativa, normatividad. 


\section{Argumentation and Uncertainty. Is a Unified Theory of Argumentation Possible?}

Abstract

The aim of this paper is to enquire on which elements are required for a unified theory of argumentation, since this might be the answer to the relativistic hints, which are accompanied by the all or nothing sophistry. It is proposed to consider various elements, such as agent, action, consequences of action, context and the concept of 'practice'.

Keywords: Argumentation, Argumentative Agent, Argumentative Practice, Carlos Pereda, Normativity. 


\section{Incertidumbre y pluralidad argumentativa}

Nuestros conceptos de "razón" y "racionalidad" han sufrido duros reveses: los escépticos, dogmáticos, subjetivistas, relativistas, posmodernos, racionalistas..., han caído en el fango de la "sofística del todo o nada”. O bien la razón cumple con los estándares más estrictos, con las más altas exigencias que nuestra mente logre imaginar o desear, o bien debemos prescindir por completo de la razón. Recordarán con seguridad la dura imposición cartesiana a nuestra racionalidad al inicio de la primera de las Meditaciones:

He advertido hace ya algún tiempo que, desde mi más temprana edad, había admitido como verdaderas muchas opiniones falsas, y que lo edificado después sobre cimientos tan poco sólidos tenía que ser por fuerza muy dudoso e incierto; de suerte que me era preciso emprender seriamente, una vez en la vida, la tarea de deshacerme de todas las opiniones a las que hasta entonces había dado crédito, y empezar todo de nuevo desde los fundamentos, si quería establecer algo firme y constante en las ciencias [...] Ahora bien, para cumplir tal designio, no me será necesario probar que son todas falsas, lo que acaso no conseguiría nunca; sino que, por cuanto la razón me persuade desde el principio para que no dé más crédito a las cosas no enteramente ciertas e indudables que a las manifiestamente falsas, me bastará para rechazarlas todas con encontrar en cada una el más pequeño motivo de duda (AT VII, 17-18; énfasis añadido).

Así, Descartes pensó que bastaba encontrar un mínimo atisbo de duda en cualquiera de sus creencias para abandonarla: i.e., una condición suficiente para rechazar una creencia como no fundada era encontrar una posibilidad lógica que le hiciera dudar de ella. La posibilidad de que nuestros sentidos nos engañen, de que estemos soñando, o de que un genio maligno nos esté timando, fueron un imaginario lógico suficiente para que Descartes rechazara todas sus creencias que tuvieran como fuente la percepción sensorial o el uso de su razón. Fueron tan altas las exigencias a las que Descartes 
sometió a la racionalidad humana que pronto se quedó solo, encerrado en sus pensamientos, en el más cruel de los solipsismos, del que sólo pudo sacarlo su dios benevolente.

El patetismo de la "sofística de la irracionalidad" a la que condujo "la sofística del todo o nada”, es expresado sin recato por Schelling en sus Investigaciones filosóficas sobre la esencia de la libertad humana:

Ésta es la tristeza inherente a toda vida finita, e incluso si hay en Dios una condición al menos relativamente independiente, es también una fuente de tristeza, que, empero, nunca se hace efectiva, sino que sólo está al servicio de la eterna alegría de la superación. De ahí el velo de tristeza que se extiende sobre toda la naturaleza, la profunda e inquebrantable melancolía de toda vida (VII, 399).

Para Steiner, Schelling atribuye a la existencia humana una pesadumbre (Schwermut) ineludible, una tristeza en la que se apoyan tanto la conciencia como el conocimiento, y que es el fundamento de toda percepción y proceso mental (Steiner, 2005: p. 11). Así, la sofística de la irracionalidad, dadas las exigencias planteadas a la razón humana, termina por volver sospechosa a la razón misma.

También contemplamos la estela del cartesianismo a lo largo y ancho de la Modernidad. Se encuentra en el pensamiento matematizante lebniziano, y en sus paradojas sobre la libertad humana; en el intento geométrico de Spinoza por fundamentar la ética; en las distinciones empiristas entre el conocimiento a priori y a posteriori, $\mathrm{y}$ entre enunciados y juicios analíticos y sintéticos; en el escepticismo teórico de Hume, y sus críticas a la razón, y a las nociones de causalidad e inducción; en el intento kantiano de fundamentar, a través de la razón pura, a la ética; en la Aufhebung hegeliana, y sus aspiraciones de un sistema absoluto y totalizante; en el proyecto logicista de Frege y Russell; en el intento de una construcción [Aufbau] lógica del mundo de Carnap; en el emotivismo moral y estético de los positivistas lógicos; así como en la norma del "todo vale" que rige a las más variopintas especulaciones posmodernas. 
Todos estos proyectos e intentos, asumidos desde "la sofística del todo o nada", tienden a compartir un concepto común de "razón", un concepto "austero", cuyo modelo de funcionamiento es el algoritmo. Este concepto austero de "razón” es el que desencadena la no pocas veces incontenible "sofística de la irracionalidad": se piensa que o bien disponemos de una razón austera - con modelos certeros para construir y reconstruir conceptos, criterios fijos, precisos y generales para argumentar, y programas fundamentalistas de justificación-, o no nos queda más que el "todo vale" y sus versiones más enconadas: el escepticismo y el relativismo epistémicos. Estos programas racionalistas, deductivistas y fundamentalistas buscan excluir por completo a la incertidumbre, y reducirse a lo indubitable, al cálculo exacto y al respaldo necesario de conclusiones. Sin embargo, no hay por qué aceptar de inicio la alternativa del "todo o nada", pues parece que:

Encontramos racionalidad en donde encontramos argumentación, y ésta se dice de muchas maneras: hay diferentes esquemas argumentales y varias posibilidades de formular un ataque argumental o de respaldar una conclusión. Así, eliminar de la razón, como requisitos necesarios, atributos tales como "criterios precisos, fijos y generales" o "creencias últimas en tanto fundamentos”, y vincularla a la delicada aventura de los ciclos argumentales despide un concepto austero de razón, una razón cierta de sí en tanto singular, homogénea, demarcada, con relaciones exclusivamente necesarias. Pero no despide a la razón. Por el contrario, le da la bienvenida a un concepto enfático de razón e invita a vivir con su incertidumbre, a enfrentarnos sin cesar a ella (Pereda, 1994b: pp. 9-10).

Ecos de una posición que no excluye a la incertidumbre de la razón los encontramos también en la historia de las ideas: está en el pensamiento moral aristotélico que asume a la incertidumbre como un componente esencial de la vida moral, donde el hombre prudente y culto asume esa realidad, y en el que la prudencia (phrónesis) se concibe como el medio ideal para administrar dicha carencia de certezas. 
Está también en el alegato en pro del "espíritu de fineza" (esprit de finesse) pascaliano, y en su oposición al "espíritu geométrico" (esprit de géométrie). Y, en Kant, se aloja en el concepto de "juicio reflexionante" (reflektierenden Urteilskraft), con el que deseaba mostrar que el ser humano es un racionalizador vagabundo, y lo ejemplificaba perfectamente en las particularidades de nuestros juicios estéticos y el libre juego de nuestras facultades. Carlos Pereda recuperó estas intuiciones bajo el concepto de "razón enfática": aquélla que admite el lenguaje figurado, la probabilidad, que toma en cuenta la historia de los conceptos y de los términos, y que considera relevante quién dice una cosa y a quién la dice.

De este modo, no resultará extraño que se pueda afirmar hoy sin vaguedades que la defensa de "la incertidumbre de la razón [es] en la mejor defensa de la razón: la defensa de una razón enfática pero sin falsas ilusiones de seguridad y, por eso mismo, sin desengaños suicidas" (Pereda, 2004: pp. 11-12). Y también que, en la actualidad, la argumentación ya es el nuevo paradigma de la razón humana, lo que quizá constituya una de las mayores revoluciones dentro del pensamiento occidental (Jales Ribeiro, 2012: p. 11).

\section{Hacia una teoría unificada de la argumentación}

Con la argumentación sucedió algo muy similar a lo que sucedió con nuestro concepto de "razón”: se pensó que “o la argumentación es singular, se reduce a la deducción, en general, a un algoritmo, o no es más que un plural de formulaciones verbales regidas por varios deseos (deseos de expresarse deseos de estar con otros, deseos de pasar el tiempo de algún modo...) y nada más" (Pereda, 1994b: p. 10). De ser así, la mayoría de los casos que solemos considerar como argumentación no lo serían. Por ejemplo, nuestras argumentaciones entre amigos, entre familia, así como nuestras argumentaciones morales y políticas, e incluso gran parte de las argumentaciones científicas no poseerían un adecuado respaldo racional. Todo ello se reduciría, para quien sucumbe nuevamente ante la "sofística del todo 
o nada”, a simple retórica; entendiendo por ésta la decoración o el revestimiento verbal guiados por el azar.

Quizá sea esto lo que explique la negligencia histórica y filosófica con respecto a la teoría de la argumentación. Algo que no sucedió con el desarrollo de la lógica formal. Es por ello que la teoría de la argumentación es de cuño reciente. No fue hasta a mediados del siglo pasado, con la aparición en 1958 de The Uses of Argument de Stephen Toulmin y el Traité de l'Argumentation de Perelman y OlbrechtsTyteca, que los estudios sobre nuestros intercambios argumentativos ordinarios recibieron una atención independiente. Merecen también la atención los trabajos de Hamblin (1970), Johnson (2000), Walton (2006), y van Eemeren y Grootendorst (1992).

La principal pregunta que buscan responder los teóricos de la argumentación es de índole normativa: ¿qué hace que un argumento sea un buen argumento? Para ello, se han tratado de desarrollar teorías lo más completas posibles que permitan, o capturar la normatividad de nuestros intercambios argumentativos ordinarios, o establecer ciertos criterios sobre los cuales se deban construir nuestras argumentaciones. Aquí radica ya una primera división entre las distintas teorías de la argumentación: algunos teóricos se concentran en el carácter normativo de la teoría de la argumentación, centrada en la evaluación de argumentos; y otros en el carácter normativo de la práctica argumentativa, pues el argumentar presupone normas. Lo que buscan las tendencias más recientes es dar cuenta de la primera a través de la segunda.

Sin embargo, el problema es añejo. Aristóteles mismo ya se había percatado que en nuestro natural y ordinario ejercicio de dar y recibir razones se traslapan distintas dimensiones del argumento: logos, ethos y pathos. Para ello diseñó distintas herramientas que dieran cuenta de dichas dimensiones: la lógica, la dialéctica y la retórica (Berti, 1989). Para el aristotelismo posterior, la pluralidad metodológica aristotélica, que toma en cuenta tanto la naturaleza del objeto de discusión como los intereses teóricos y prácticos de los participantes en el debate, está garantizada por la falta de reduccionismo con la que el Estagirita pensó al ser humano: pues éste no es sólo razón, y los recursos de la razón no agotan la totalidad de los 
recursos del hombre. Así, "buena parte de los avances en la capacidad dialógica consisten en un progresivo ensanchamiento del horizonte, que de ordinario supone la superación de frenos de carácter racionalista: la inteligencia humana no es sólo razón, es también intelecto; para persuadir no basta razonar bien, pues también hay que infundir confianza y establecer sintonía emotiva [...]; [y] el lenguaje no es sólo semántica sino también pragmática" (Jiménez Cataño, 2006: p. 12). Aristóteles lo sabía: la verdad no basta, tampoco el mejor argumento visto desde una estricta dimensión lógica, pues al menos son débiles en dos sentidos: (a) es posible tener la verdad, y es posible disponer del mejor argumento, y no hacerlo valer (¿cuántas veces no hemos sufrido el hecho de tener la razón y que no nos crean o no logramos convencer a nuestro interlocutor?); y (b) incluso con la verdad y con los mejores argumentos, también se puede maleducar, desinformar y corromper.

En la actualidad, dicho problema persiste: se trata de brindar una teoría sistemática que responda por la pregunta normativa que nos hace considerar algunos argumentos como buenos y otros como malos, pero sin caer en un enfoque reductivo. Este problema fue bautizado por Johnson como "el problema de la integración” (2000: p. 191). Sin embargo, los teóricos de la argumentación se han concentrado en uno u otro de los enfoques, tratando de responder a la pregunta normativa desde uno solo, creyendo que es posible reducir la normatividad de todas las dimensiones del argumento a una sola.

Por ejemplo, el clásico Introduction to Logic (1953) de Copi se concentró en la dimensión lógica del argumento. Algunas de las críticas más habituales a las que ha sido sometido el enfoque de Copi tratan de hacer ver la necesidad de concebir a nuestros argumentos como situados; i.e., la buena o mala argumentación, para poderla considerar de uno u otro modo, tiene una alta dependencia contextual. Por lo mismo, aunque Copi avanza en el análisis de la dimensión lógica de nuestras argumentaciones, muchas de las herramientas que nos brinda son limitadas para el análisis de nuestros intercambios argumentativos ordinarios. Algunas de las falacias tipificadas por Copi no es fácil considerarlas como tales en determinados contextos: e.g., una falacia bien puede tener un alto poder retórico, una falacia de relevancia 
bien puede no ser tal considerada en el contexto dialógico, o bien muchos de los esquemas argumentativos que nos permiten clasificar falacias son los mismos que funcionan para argumentos no falaces.

Algo similar sucede cuando nos concentramos ya sea en la dimensión dialéctica - como lo hizo la escuela pragma-dialéctica de van Eemeren y Grootendorst- o en la retórica -como lo hicieran originalmente Perelman y Olbrechts-Tyteca (1958), y más recientemente Tindale y Gilbert (1997)-. Por ello, quizá una de las tareas necesarias de la teoría de la argumentación hoy siga consistiendo en brindar una teoría no reductiva que permita responder a la pregunta normativa.

Se han dado recientes pasos en este camino al considerar a la argumentación desde el punto de vista de la pragmática: como un acto de habla (speech act). Uno de los últimos resultados dados por esta vía es Giving Reasons (2011) de Lilian Bermejo-Luque. En esta aproximación reciente, la autora busca desarrollar una teoría de la argumentación que permita responder a las cuestiones normativas sin caer en una posición reductiva. Para ello, Bermejo-Luque sugiere considerar a la argumentación como un acto de habla complejo, lo cual nos permitiría responder, desde un punto de vista pragmalingüístico, a la pregunta normativa.

Para Bermejo-Luque, el primer paso consiste en considerar a la argumentación principalmente como una actividad lingüística. Con ello, la autora busca distinguir su postura de aquellas que consideran la argumentación como un producto lógico, un procedimiento dialéctico, un proceso retórico o como una herramienta epistemológica. En un segundo momento, y siguiendo a van Eemeren y Grootendorst, considera la argumentación como un complejo acto de habla, lo que nos permitiría - piensa- integrar las distintas dimensiones de la argumentación en una teoría sistemática y unitaria.

Bermejo-Luque sugiere que la teoría de la argumentación ha carecido de un tratamiento unitario de los dos aspectos centrales de nuestras prácticas argumentativas: su poder de justificación y su poder persuasivo. Las teorías más recientes, incluso, tienden a considerar la argumentación, ya sea como un dispositivo útil para la justificación, que eventualmente puede ser usado para persuadir; o bien como un dispositivo persuasivo, cuyas condiciones de legitimidad 
pueden dar cuenta de la justificación. Para Bermejo-Luque, considerar la argumentación como un acto de habla complejo nos proporcionaría dicho tratamiento unitario de la justificación y la persuasión razonable de nuestras argumentaciones: así, la argumentación sería un dispositivo útil para la justificación en su nivel ilocucionario, mientras que su poder persuasivo resultaría de su capacidad para producir ciertos efectos perlocucionarios. ${ }^{1}$

Bermejo-Luque aborda la argumentación de manera muy general como una actividad humana, incluso como el paradigma de nuestros intercambios lingüísticos (siguiendo la teoría inferencialista de Brandom, y la teoría de la acción comunicativa de Habermas). Realiza también un rastreo histórico de la negligencia que durante siglos sufrió el estudio de nuestras argumentaciones cotidianas, y atribuye dicha negligencia a la recepción histórica y sesgada de Aristóteles. En un segundo momento, reconstruye los debates recientes en teoría de la argumentación, y repasa algunos de los problemas principales que surgen cuando adoptamos una teoría reductiva. Por último, aborda algunos problemas meta-teóricos para defender la aproximación pragma-lingüística a la argumentación. En particular, defiende que una teoría de la argumentación desde un enfoque pragma-lingüístico evita el instrumentalismo en el que caen las teorías reductivas rivales. Si no evitamos el instrumentalismo - nos dice Bermejo-Luqueuna teoría normativa de la argumentación es incapaz de superar el problema de la justificación al que se enfrenta.

Ahora bien, ¿qué sucede si caracterizamos a la argumentación como un acto de habla complejo? Desde esta perspectiva, el acto de habla de argumentar cuenta como un intento de mostrar una afirmación como correcta. De este modo, un buen argumento estaría definido como el que de hecho muestra que la afirmación que defiende

1 Para comprender mejor qué es un efecto perlocucionario, véase Searle, 1969: p. 34: "[...] correlativamente a la noción de actos ilocucionarios está la noción de las consecuencias o efectos que tales actos tienen sobre las acciones, pensamientos o creencias, etc., de los oyentes. Por ejemplo, mediante una argumentación yo puedo persuadir o convencer a alguien, al aconsejarle puedo asustarle o alarmarle, al hacer una petición puedo lograr que él haga algo, al informarle puedo convencerle (instruirle, elevarle -espiritualmente-, inspirarle, lograr que se dé cuenta)". 
es correcta, lo cual también nos da cuenta de la justificación. Los argumentos serían objetos abstractos que representan propiedades semánticas en los actos de argumentar.

No obstante, una perspectiva que trate de brindar una teoría unificada de la argumentación desde los actos de habla no carece de dificultades. Lo que ahora nos dicen los lingüistas y los filósofos del lenguaje es que formular una teoría de los actos de habla es mucho más difícil de lo que parecía inicialmente. Cuando se estudia la fuerza ilocucionaria de los actos de habla, la mayoría de las veces se llega a la conclusión de que el agente lingüístico carece de control epistémico sobre el significado de sus palabras; i.e., el interlocutor puede tomarlas en última instancia como él quiera, sin que el hablante pueda lograr que sus palabras se tomen como él hubiese deseado. Esta posición, que cada día gana más adeptos, suele ser llamada "externismo ilocucionario" (Blanco Salgueiro, 2004). El problema es el siguiente: si el externismo ilocucionario es verdadero, entonces una teoría de la argumentación sistemática desde los actos de habla es imposible. El agente argumentativo, como el agente lingüístico, carecería de control epistémico sobre sus argumentos, lo cual terminaría por diluir cualquier esperanza de control sobre la normatividad de nuestros intercambios argumentativos. ${ }^{2}$ Así, aunque una caracterización descriptiva de la argumentación como acto de habla puede parecer sugerente, como teoría normativa puede fracasar si el externismo ilocucionario es verdadero. O bien, no lograría capturar la normatividad de la argumentación, o terminaría en un escepticismo o relativismo sobre nuestros intercambios argumentativos.

Entonces, ¿qué aspectos debe tomar en cuenta una teoría de la argumentación unificada? En primer lugar, habría que concebir a la argumentación como un tipo de "acción". Así, para cualquier acción, hay tres elementos que podrían ser considerados como

2 Explicito el argumento: $\mathrm{P}_{1}$. No le es posible al hablante determinar por completo la fuerza ilocucionaria de cualquiera de sus actos de habla; $P_{2}$ (implícita). Si el hablante no puede determinar por completo la fuerza ilocucionaria de sus actos de habla, menos aún podrá tener control epistémico completo sobre su fuerza ilocucionaria; P3. (implícita). Un argumento es un acto de habla; C. Por tanto, el hablante no tendrá control epistémico sobre la fuerza ilocucionaria de sus argumentos. 
normativamente interesantes: primero, el agente, la persona que realiza el acto; en segundo lugar, la acción en sí misma; en tercer lugar, las consecuencias de la acción. De este modo, se han agrupado históricamente a los tres grandes sistemas éticos -las teorías de la virtud, las teorías deontológicas y las teorías consecuencialistas-, dado que cada una enfatiza uno de estos elementos. De igual modo, podríamos decir que una teoría de la argumentación puede concentrarse en cualquiera de estos elementos. O si aspira a unificar todos los aspectos de nuestros intercambios argumentativos, debería concentrarse, inicialmente, en los tres elementos.

En primer lugar, una teoría unificada de la argumentación debe considerar al agente argumentativo: lo que daría lugar a una teoría de las virtudes argumentativas. Por lo anterior, quizá no debería sorprendernos que los grandes avances que se han logrado en ética y en epistemología a partir de la teoría de las virtudes - en las obras de Anscombe, Foot, Sosa, Williams, McDowell, etc.- pudiera trasladarse a la teoría de la argumentación, resolviendo sus problemas normativos, al igual que pretende hacerlo con cuestiones morales y epistémicas. Desde esta perspectiva, cuando una argumentación fracasa debe considerarse la posibilidad de que la causa sea una falla de habilidad en quien argumenta: un fallo de virtud. Recientemente, Andrew Aberdein (2010) ha propuesto una tipología de las virtudes argumentativas: (a) respecto a la disposición a participar en la argumentación, quien argumenta: debe ser comunicativo, tener fe en la razón, poseer coraje intelectual y sentido del deber; (2) respecto a la disposición para escuchar a los demás, quien argumenta: debe tener empatía intelectual, comprensión de las personas, los problemas y las teorías, debe ser justo de pensamiento, equitativo en la evaluación de los argumentos de los demás, debe tener amplitud en la recopilación y en la evaluación de las pruebas, debe ser capaz de reconocer a la autoridad confiable y a los hechos relevantes, y debe ser sensible a los detalles; (c) con respecto a la voluntad de modificar la propia posición, quien argumenta: debe tener sentido común, humildad e integridad intelectuales, honor, responsabilidad y sinceridad; y (d) respecto a la voluntad de cuestionar lo obvio, quien argumenta: debe tener un adecuado respeto a la opinión pública, debe ser autónomo, 
perseverante, diligente, cuidadoso y riguroso. Por mi parte, he considerado en otro lugar (Gensollen, 2012) que al menos hay cinco virtudes argumentativas básicas, que son condiciones necesarias para que la argumentación siga un curso exitoso: buena voluntad, caridad, falibilismo, coalescencia, cosmopolitismo y pluralismo.

En segundo lugar, una teoría unificada de la argumentación debe considerar al argumento en sí mismo: quizá el problema central de la lógica haya sido el estudio de la validez formal, así como el de la filosofía de la lógica la caracterización de los conceptos de "validez" y de "consecuencia lógica". Una de las tareas más acuciantes dentro de la teoría de la argumentación contemporánea consiste en establecer si es posible caracterizar una noción de "validez no formal", dado que el concepto mismo de "validez" fue establecido desde los lenguajes formales. En este contexto, Toulmin (1958) proporcionó herramientas para analizar lógicamente la argumentación que se realiza en el lenguaje natural, y defendió una teoría no formal -aunque de cierta forma sistemática- de la inferencia.

En tercer lugar, una teoría unificada de la argumentación debe considerar los efectos o consecuencias de la argumentación (lo que los pragmatistas llaman "efectos perlocucionarios"). Aquí se concentran las distintas dimensiones de la argumentación: la lógica, la dialéctica y la retórica. Desde la dimensión lógica se deben considerar a los argumentos como dispositivos de justificación de creencias: así, los vínculos entre la lógica, la epistemología y la argumentación se iluminan correctamente. Algunos, incluso, piensan la teoría de la argumentación como una especie de "epistemología aplicada" (Bermejo-Luque, 2009). Desde la dimensión lógica, por tanto, los argumentos son productos que buscan la verdad, que aspiran a la validez, y que justifican creencias. ${ }^{3}$ Por su parte, desde la dimensión dialéctica, los argumentos son procedimientos de dar y recibir razones

3 Las reglas inferenciales son independientes -hasta cierto punto- de las reglas procedimentales y morfológicas de la argumentación; i.e., podemos juzgar si una inferencia es válida o no hasta cierto punto de manera independiente desde la dimensión lógica, no teniendo en cuenta la dimensión dialéctica y retórica de la argumentación. No obstante, como se infiere de lo anterior, las reglas inferenciales no son las únicas reglas presentes en nuestras prácticas argumentativas (Pereda, 1994: cap. 1). 
que buscan resolver conflictos de creencias. Desde esta perspectiva, estaremos interesados en estudiar las diferencias de opinión, el desacuerdo y la discusión argumentativa, la diferencia entre diferencias de opinión implícitas y explícitas, los tipos de diferencias de opinión (diferencias de opinión principales y subordinadas), el cómo reconocer puntos de vista y dudas, así como los distintos indicadores de la argumentación contenidos en el lenguaje natural. Por último, desde la dimensión retórica, consideraremos los argumentos como actos comunicativos que buscan persuadir: esto lo podemos hacer ya sea desde el esquema tradicional de la inventio, dispositio, elocutio, memoria y actio; o bien podemos considerar, siguiendo a Toulmin, que los argumentos sustantivos son dependientes de los diversos campos argumentativos (algo similar a lo que Aristóteles tuvo en mente en su Retórica cuando consideró la importancia de la audiencia).

En cuarto y último lugar, una teoría unificada de la argumentación debe considerar la alta dependencia contextual que tienen nuestros intercambios argumentativos. Todo ciclo argumentativo siempre se da en medio: parte de un terreno común que comparten las partes en conflicto (presupone un lenguaje común, creencias, intereses, etc.), y-si llega a buen puerto- termina presumiendo al menos algunos contenidos proposicionales que es posible inferir a partir de los contenidos proposicionales a los que se ha llegado por medio de la argumentación. Para siquiera poder iniciar un ciclo argumentativo, una condición necesaria es que las partes en conflicto compartan un sinnúmero de creencias (entre otras muchas cosas). Las creencias en disputa se dan sobre una sólida y vasta meseta de creencias compartidas. Sin ellas, no sería posible evaluar otras creencias, ni las razones a favor o en contra de ellas. Esto suele ser denominado por pragmatistas y teóricos de la argumentación como "terreno común" (common ground) (Stalnaker, 2002). Además, toda argumentación, cuando termina, presume algunas consecuencias de lo acordado. Presume algunos contenidos proposicionales que es posible inferir (a veces gradualmente) de los contenidos proposicionales en los que se expresa el acuerdo: por ello, es posible evaluar argumentos no sólo por lo que expresan explícitamente, sino por alguna o varias de sus consecuencias. También, toda argumentación presume alguna 
o algunas acciones que se siguen del acuerdo: e.g., actuar como si $p$ fuese verdadero, deseable, correcto, adecuado (siendo $p$ el contenido proposicional del acuerdo al que se ha llegado). Así, toda argumentación está situada: inicia necesariamente con presuposiciones acerca de creencias, deseos, intereses de las partes en conflicto; y termina presumiendo contenidos proposicionales y acciones que se siguen de los acuerdos a los que han llegado las partes.

El esquema de los elementos necesarios para una evaluación de la argumentación queda ahora completo, partiendo de la analogía con la evaluación de las acciones en general:

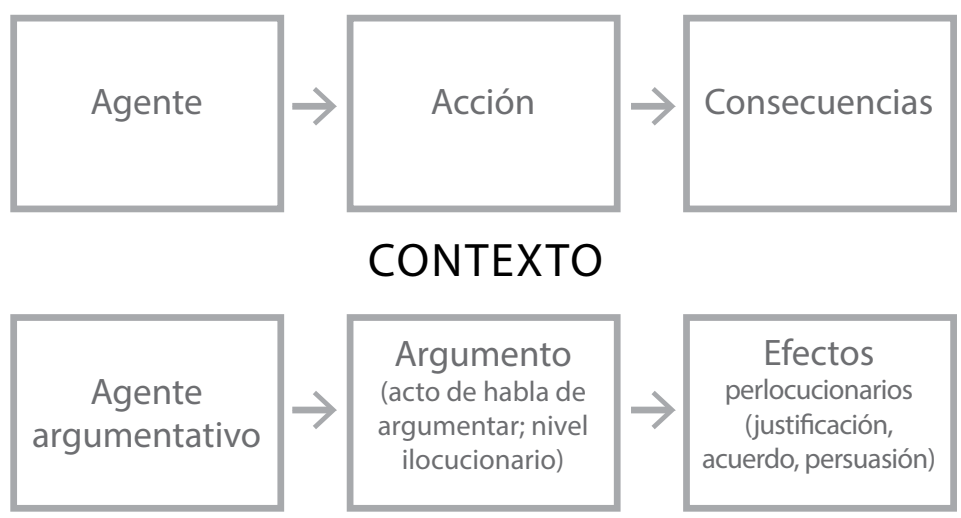

Ahora bien, ¿a dónde llegamos al considerar tanto al agente argumentativo, a los argumentos y sus consecuencias, así como al contexto argumentativo? Hasta ahora sólo he señalado todos los elementos que es necesario tomar en cuenta para buscar una teoría unificada de la argumentación, pero no he señalado ningún elemento unificador.

\section{Argumentación en cuanto práctica}

Quizá el concepto que integre los diversos elementos que deben considerarse en una teoría unificada de la argumentación sea el de "práctica". Así lo intuye Carlos Pereda en un texto muy reciente: 
"Propongo que indagar en qué consiste argumentar en cuanto práctica (y no, por ejemplo, en cuanto diversas conexiones entre enunciados, o en cuanto un tipo de acto de habla) es adoptar la perspectiva más rica y más abarcadora sobre el argumentar" (Pereda, 2010: p. 47). Siguiendo esta línea, atenderé una serie de sugerencias e insinuaciones a este respecto, más que con los esbozos de una posible teoría.

En primera instancia, podemos decir que son condiciones necesarias del concepto de "práctica" las siguientes: (1) uno o varios “agentes”, ya sea individuales o colectivos; (2) los "propósitos" constitutivos de dicha práctica, y los propósitos particulares de quien o quienes la realizan; (3) los "medios" tanto internos como externos de los que se echa mano para cumplir con los propósitos; (4) los "modos" de una práctica, que surgen de la interrelación de los propósitos y los medios; y (5) los "recursos" de los que dependen los medios, y que a su vez dependen de contextos naturales y sociales.

A partir de estas condiciones -agentes, propósitos, medios, modos y recursos- quizá sea posible incorporar los elementos mencionados previamente. Como materiales internos, tendríamos a las condiciones (2), (3) y (4). Con respecto a la segunda-los propósitos-, podemos conformar el siguiente entorno conceptual que atiende al elemento de las consecuencias de nuestras argumentaciones: convencer, convencerse, deslumbrar, dominar, hacer patente, modificar creencias, persuadir, resolver conflictos, tratar problemas, etc. Con respecto a la tercera condición, los propósitos se logran a través de ciertos medios. De ahí un nuevo entorno conceptual que puede incluir: abducción, atacar propuestas, apoyar creencias, concluir, deducción, inducción, inferir, inferencias, premisas, problemas, etc. Estos medios pueden ser externos o internos. Los externos atienden a los elementos que constituyen lo que llamamos el "contexto argumentativo" (al menos a una parte de éste): al terreno común de la argumentación y a las presunciones que quedan después de terminado el ciclo argumentativo. Por su parte, los medios internos están articulados por una conexión de enunciados que vinculan las premisas a una conclusión, lo cuales recupera el elemento del argumento en sí mismo. Un último material interno nos lo da la cuarta condición, o los modos, de lo que surge un último entorno conceptual integrado por: alegato, conversar, 
debate, dialogar, discrepar, discutir, impugnar, meditar, reflexionar, etc. Esta condición también recupera partes importantes del contexto argumentativo, pues no siempre se argumenta del mismo modo (ni bajo las mismas reglas).

Como materiales externos, tenemos a la primera y a la quinta condiciones: al agente y a los recursos. Como ya dijimos, una teoría de las virtudes argumentativas recupera los aspectos relevantes del agente en las prácticas argumentativas, no obstante que las relaciones entre los agentes y las prácticas suelen ser demasiado complejas. Con respecto a la quinta condición, los agentes cuentan con diversos recursos tanto físicos como psicológicos que, al menos en parte, dependen de su herencia natural. No obstante, a un agente también lo condiciona su herencia cultural articulada en su historia pasada y en su medio circundante. La relación entre un agente y sus recursos es bastante compleja, pues el agente se conforma condicionado por una gran cantidad de recursos que el agente mismo rehace en parte. Esta última condición, como puede verse, integra aún más aspectos del contexto argumentativo.

Así, una teoría de la argumentación desde las prácticas argumentativas parece integrar todos los aspectos y elementos relevantes para tratar de articular una respuesta a la pregunta normativa inicial: ¿qué hace de un argumento un buen argumento? Sin embargo, la tarea para los teóricos de la argumentación aún sigue pendiente.

\section{Conclusión}

Concluyo. Si oponemos un concepto de "razón enfática” al concepto moderno de "razón austera", y también oponemos un concepto plural de argumentación al monismo deductivista, entonces se genera un vínculo necesario entre racionalidad y argumentación. El ejercicio de la razón no sería, entonces, otra cosa que la tarea epistémica de dar argumentos de varias clases y en varios niveles, siempre dependiendo del objeto de estudio y de debate, y de nuestros intereses tanto teóricos como prácticos. 
Así, la teoría de la argumentación -como un trabajo multidisciplinario en el que confluyen las ciencias de la comunicación, la lingüística, la semántica, la pragmática, la lógica informal y formal, la lógica dialéctica, la pragma-dialéctica, la psicología, la epistemología, la filosofía de la ciencia y las ciencias cognitivas-- puede tener ya un lugar adecuado dentro del panorama del conocimiento humano; y uno sin duda muy importante. No obstante, es una disciplina joven y en transición. He propuesto que debemos considerar diversos elementos si deseamos una teoría unificada de la argumentación: al agente argumentativo, a los argumentos considerados en sí mismos, a los efectos diversos que tienen nuestras argumentaciones, así como al contexto argumentativo. También he propuesto que el concepto de "práctica" puede ser el elemento unificador que nos lleve en un futuro a contar con una teoría unificada de la argumentación. Pero el trabajo, como en casi cualquier disciplina cognitiva nueva, está aún por hacerse.

Agradezco a Francisco Ramírez y a Marc Jiménez Rolland su ayuda en la redacción de este texto, y sus atinados comentarios.

\section{REFERENCIAS BIBLIOGRÁFICAS:}

Aberdein, Andrew. 2010. "Virtue in Argument" en Argumentation, Vol. XXIV, No. 2, pp. 165-179.

Bermejo-LuQue, Lilian. 2011. Giving Reasons. A Linguistic-Pragmatic Approach to Argumentation Theory. Dordrecht: Springer.

Bermejo-Luque, Lilian. 2009. "Argumentation Theory and the Conception of Epistemic Justification” en Studies in Logic, Grammar and Rhetoric, Vol. 16, No. 29, pp. 285-303.

BerTI, Enrico. 1989. Las razones de Aristóteles. Tr. Horacio Gianneschi y Maximiliano Monteverdi. Buenos Aires: Oinos, 2008.

Blanco Salgueiro, Antonio. 2004. Palabras al viento: ensayo sobre la fuerza ilocucionaria. Madrid: Editorial Trotta.

CopI, Irving M. 1953. Introduction to Logic. New York: MacMillan. 
Descartes, René. 1641. Meditaciones metafísicas con objeciones y respuestas. Tr. Vidal Peña. Madrid: Alfaguara, 1977.

Gensollen, Mario. 2012. "Virtudes argumentativas: hacia una cultura de la paz", en Euphyía, Vol.VI, No. 11, pp. 115-131.

Gilbert, Michael A. 1997. Coalescent Argumentation, Routledge, Mass:York University.

Hamblin, C.L. 1970. Fallacies. London: Barnes \& Noble Inc.

Jales Ribeiro, Henrique (ed). (2012). Inside Arguments. Logic and the Study of Argumentation. New Castle: Cambridge Scholars Publishing.

Jiménez Cataño, Rafael. 2006. "Mi verdad, tu verdad” en Sýnesis. Cuadernos de Humanismo Contemporáneo, No. 1. México: Los Libros de Homero \& Universidad Bonaterra.

Johnson, Ralph H. 2000. Manifest Rationality: A Pragmatic Study of Argument. Mahwah, NJ: Lawrence Erlbaum Associates.

Pereda, Carlos. 1994a. Vértigos argumentales: una ética de la disputa. México Anthropos.

Pereda, Carlos. 1994b. Razón e incertidumbre. México: UNAM \& Siglo XXI.

Pereda, Carlos. 2010. "La argumentación en cuanto práctica" en Carretero, F. \& Ramírez, C. \& Favila, V. (coords.), Introducción a la teoría de la argumentación (pp. 47-60). Guadalajara: Universidad de Guadalajara.

Perelman y Olbrechts-Tyteca. 1958. Traité de l'argumentation. La nouvelle rhétorique. Bruxelles: Éditions de Université libre de Bruxelles.

Schelling, F.W.J. 1809. Investigaciones filosóficas sobre la esencia de la libertad humana y los objetos con ella relacionados. Tr. Helena Cortés y Arturo Leyte. Madrid: Anthropos, 1989.

Searle, John. 1969. Actos de habla. Tr. Luis M. Valdés Villanueva. Madrid: Cátedra, 1990.

Stalnaker, R. 2002. "Common Ground" en Linguistics and Philosophy, Vol. 25, No. 5-6, pp. 701-721.

Steiner, George. 2005. Diez (posibles) razones para la tristeza del pensamiento. Tr. María Condor. México: Fondo de Cultura Económica \& Siruela, 2007.

Toulmin, Stephen. 1958. The Uses of Argument. New York: Cambridge University Press, 2003.

van Eemeren \& Robert Grootendorst. 1992. Argumentation, Communication, and Fallacies: A Pragma-dialectical Perspective. London: Routledge.

Walton, Douglas. 2006. Fundamentals of Critical Argumentation. Cambridge, Mass: Cambridge University Press. 